\title{
Progress in Chemical Constituents and Pharmacological Effects of Lindera glauca
}

\author{
Jianchen Liu, Chunyan Du, Yanan Li, Shiman Zuo, Rui Tan, and Hezhong Jiang* \\ School of Life Science and Engineering, Southwest Jiaotong University, Chengdu, Sichuan 611756, China \\ Email: jianghz10@home.swjtu.edu.cn (H. J.)
}

\begin{abstract}
Lindera glauca is a folk medicine with rich resources in China. Previous phytochemical studies on L. glauca have resulted in the isolation and identification of the main chemical constituents such as alkaloids, sitosterol, flavonoids, sesquiterpenes, and lignans, as well as essential oil, with antimicrobial, antitumor activity and other pharmacological effects. In this paper, the chemical compositions and pharmacological effects of $L$. glauca were summaried.
\end{abstract}

Keywords Lindera glauca, chemical constituents, pharmacological effects

\section{Introduction}

Lindera glauca, belonging to the Lindera genus (Lauraceae), is a deciduous shurb or small arbor plant with rich resources in China, distributed mainly in Zhejiang, Jiangxi, Anhui, Hunan, Sichuan, Yunnan, and other provinces. L. glauca was used as folk medicine to treat stroke, rheumatism and other diseases in China. In modern research, it is also widely used in the fields of food, medicine, chemical industry, and so on, ${ }^{[1,2]}$ which attracted a lot of researchers to investigate this plant. Herein, we summarized the chemical constituents and pharmacological studies of $L$. glauca.

\section{Chemical constituents of $L$. glauca}

Previous phytochemical investigations have been carried on the chemical compositions of the various parts of $L$. glauca, and the main compositions currently reported including essential oil, alkaloids, steroidal compounds, flavonoids, and sesquiterpenoids. Wheras, the content of chemical compositions are different in different parts of $L$. glauca.

\section{Essential oil}

Lauraceae is famous as perfume plant resources throughout the world. Thus the essential oil of the Lauraceae plants is researched and applied by many scholars and companies. Volatile oil in L. glauca is mainly found in fruits, leaves and flowers. The main compositions from $L$. glauca fruit contains $\beta$-myrcene, $D$-limonene, eucalyptol, citronellal, 3,7-dimethyl2,6-octadiena, 3,7-dimethyl-1,6-octadien-3-ol, $\beta$-caryophyllene, et $a{ }^{\left[{ }^{[3]}\right.}$ The main components of the volatile oil from the leaves of $L$. glauca are $\beta$-phellandrene, myrcene, cycloprop, $\gamma$-cadinene, et al. ${ }^{[4]}$ The main components of the volatile oil from the flowers of $L$. glauca include sabinene, $\alpha$-myrcene, terpineol, 8-heptadecene, eucalyptol, et al. ${ }^{[5]} \mathrm{Sun}^{[6]}$ used HS-SPME and GC-MS technology to analyze the volatile components of different parts of $L$. glauca, which indicated that the volatile components of leaves and fruits of $L$. glauca were different. The content of copaene and $\alpha$-caryophyllene in the fruits was significantly higher than that in leaves. Yang ${ }^{[7]}$ used GC-MS to analyze the chemical constituents of the volatile oil from the steam distillation and solvent extraction of $L$. glauca fruits. The former is mainly composed of $n$-capric acid, germacrene $A$, 12-alkanic acid, pamphenolol, and caryophyllene oxide. The latter is mainly composed of camphene, 3,3,6-trimethyl-2norpinene, ethyl caprate, cineole, and ocimene. In addition, there are also differences in essential oil from different habitats and seasons. ${ }^{[8]}$

\section{Alkaloids}

In 2000, Chang et al. ${ }^{[9]}$ reported 7 alkaloids isolated from $L$. glauca, including (+)-3-chloro- $N$-formylnornantenine (1), $(+)$ - $N$-formylnornantenine (2), (+)-boldine (3), (+)-norboldine (4), $(-)$-norboldine (5), lycicamine (6), tetrahydroberberine (7). Then a new alkaloid named $\mathrm{N}$-cis-sinapoyltyramine (8) was reported by Yuh-Chwen Chang next year (Figure 1). ${ }^{[10]}$ Liu $^{[11]}$ obtained magnocurarine, $\mathrm{N}$-methyl litsoeine, litsoeine, boldine, norisoboldine, and noisocorydine from $n$-butyl alcohol and ethyl acetate parts of $95 \%$ ethanol extract of $L$. glauca. $N$-methyl litsoeine exhibited strong antitumor activity.

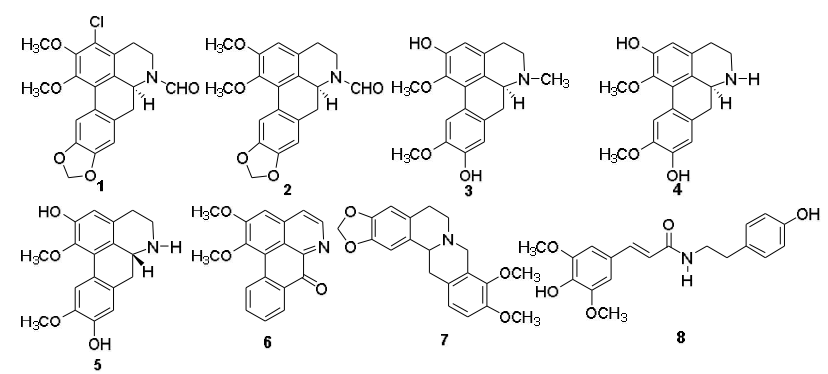

Figure 1 The structures of alkaloids isolated from L. glauca.

\section{Steroidal compounds}

In 2011, $\beta$-sitosterol (9), 7-ketositosterol (10), 7 $\beta$-hydroxysitosterosterol (11), and daucostero (12) were isolated by Gyu-Won Huh from the ethyl acetate part of $L$. glauca. ${ }^{[12]}$ Then five steroidal compounds were isolated from $L$. glauca by Yuh-Chwen Chang in 2016, named $\beta$-sitostenone (13), 
stigmasta-4,22-dien-3-one (14), 6- $\beta$-hydroxy- $\beta$-sitosternone (15), 6 $\beta$-hydroxystigmasterone (16), and $\beta$-sitosteryl- $D$-glucoside (17), repestively (Figure 2). ${ }^{[8]}$

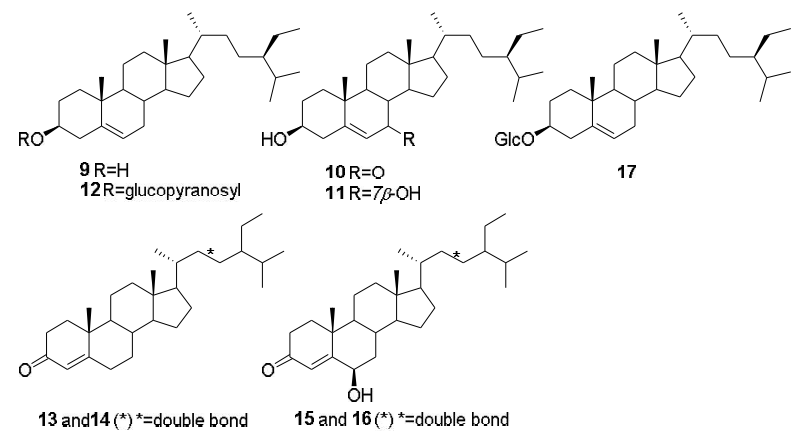

Figure 2 The structures of steroids isolated from $L$. glauca.

\section{Flavonoids}

Eight flavonoids were isolated from the heartwood of $L$. glauca by Huh et al., ${ }^{[13]}$ and were identified as lindeglaucol (18), lindeglaucone (19), cilicicone B (20), tamarixetin 3-O- $\alpha-L-$ rhamnoside (21), procysnidin A2 (22), cinnamtannin B1 (23), cinnamtannin D1 (24), and procyanidin A1 (25) (Figure 3), respectively. These flavonoids showed low density lipoprotein oxidation activity.

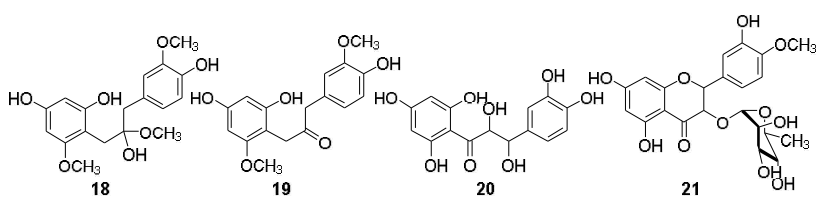

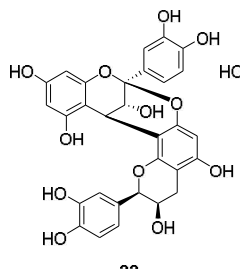
20 $\mathrm{OH}^{\mathrm{OH}}$

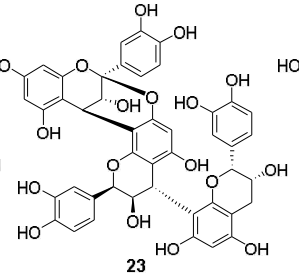

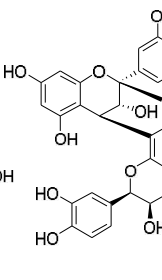
$2 \mathrm{OH}$

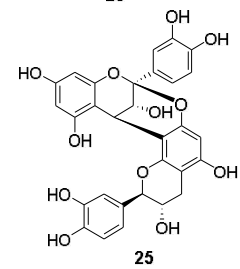

Figure 3 The structures of flavonoids isolated from L. glauca.

\section{Sesquiterpenoids}

There are four type sesquiterpenes including acyclic sesquiterpenes, monocyclic sesquiterpenes, bicyclic sesquiterpenes, and tricyclic sesquiterpenes were reported in L. glauca. The basic skeleton of acyclic sesquiterpene contained in $L$. glauca is farnesene, and its terpene is farnesene. The monocyclic sesquiterpenes are mainly curcumene, humulene, germacrene, and elemene. While the main structure of the bicyclic sesquiterpenoids in $L$. glauca is eucalyptol, juniper alkane, alliamphonoid, and irisane. The content of eucalptol (26) in $L$. glauca reached $8.1 \%$, and the content of $\gamma$-cadinene (27) in the leaves of $L$. glauca was $10.17 \%$, while the content of epishypbunol acetate in the fruits of $L$. glauca was $7.29 \%$. Cubebene (28) (Figure 4), which is tricyclic sesquiterpene, is present in the L. glauca leaves. ${ }^{[14]}$

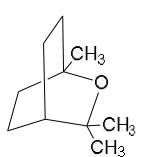

26

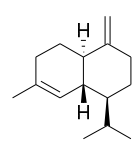

27

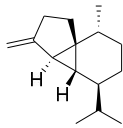

Figure 4 The structures of the main sesquiterpenoids isolated from $L$. glauca.

\section{Lignans}

Two new aryl-tetralin lignin glycosides, linderanosides A (29) and linderanosides $B(\mathbf{3 0})$, and a new dihydrobenzofuran neolignan glycoside, linderanoside C (31) (Figure 5), together with five known lignin derivatives were isolated from the trunk of the $L$. glauca by Won Se Suh in 2015. ${ }^{[15]}$

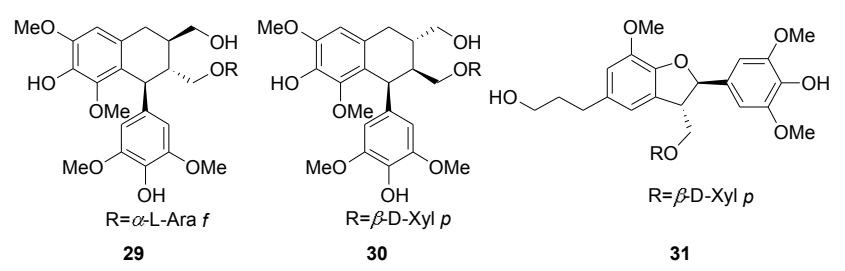

Figure 5 The structures of new lignans isolated from $L$. glauca.

\section{Other compounds}

Glaucerebroside, a new cerebroside isolated from twigs of L. glauca by Jae Sik Yu, ${ }^{[16]}$ is expected as an excellent candidate for development of novel anti-neuroinflammatory agents. In addition, butyrolactone compounds, methylparaben, phytol, squalene were found in L. glauca. Two (-)-methoxybutanolides, (3S,2E)-2-(11-dodecenylidene)-3-methoxy-4-methylenebutanoli de and (3S,2E)-2-(11-dodecynylidene)-3-methoxy-4-methylenebutanolide were isolated from the rhizomes of $L$. glauca by Katsura Seki in 1994. ${ }^{[17]}$

\section{Pharmacological reports}

The phytochemical research of $L$. glauca promoted the pharmacological investigations such as antioxidant, anti-inflammatory, anti-microbial, anti-tumor and other pharmacological effects.

\section{Antimicrobial effect}

Yang ${ }^{[8]}$ found the essential oil of $L$. glauca exhibited moderate antifungal activity. Zhang ${ }^{[18]}$ reported the volatile oil of the $L$. glauca has strong antibacterial capacity, strong inhibition of mold, and the minimum inhibitory concentration (MIC) below $0.2 \%$ for some tested bacteria. The essential oil from $L$. glauca leaves also showed various antibacterial effects on the common 14 kinds of bacteria reported by Liu in $2014 .{ }^{[5]}$

\section{Antitumor effect}

Liu $^{[11]}$ reported the $\mathrm{N}$-methyl camphor base from L. glauca exhibited strong antitumor cell proliferation activity, especially the inhibition of colon cancer cell HT-29 and gastric cancer cell SGC-7901, which was better than the positive control drug etoposide VP-16 at the medium and high concentrations. In 2011 , Wang ${ }^{[19]}$ reported that the ethanol extraction of $L$. glauca showed inhibitory activity on the chemotaxis of human breast cancer cells. 
Medicine Research

\section{Other pharmacological effects}

Zhang et al. ${ }^{[20]}$ found that the essential oil of the L. glauca leaves could relax the intestinal smooth muscle and relieve the spasm of the rabbit intestinal smooth muscle caused by ethyl phthalein. Thus the volatile oil has an obvious analgesic effect at higher dose. Cao et al. ${ }^{[21]}$ reported that the extraction of $L$. glauca had a better inhibitory activity on $\alpha$-glucosidase in yeast; moreover, the ethyl acetate fraction exhibited good inhibitory activity on $\alpha$-glucosidase in intestinal of rats. Huh ${ }^{[13]}$ reported that flavonoids from $L$. glauca were effective in the inhibition of low-density lipoprotein (LDL) oxidation in vitro.

\section{Conclusion}

The chemical constituents of $L$. glauca include mainly essential oil, alkaloids, steroids, flavonoids, sesquiterpenes, and lignans. The chemical and pharmacodynamics research also proved that $L$. glauca could be used for treatment of gastrointestinal diseases and antitumor efficacy. The alkaloids, flavonoids and butyrolactones from the genus of Lindera had been reported with excellent bioactivities. Because $L$. glauca has been used as folk medicine with rich resources in China, the further research on the chemical constituents and bioactivities should be performed.

\section{Acknowledgements}

This work was co-supported by the National Natural Science Foundation of China (Nos. 81473337, 31200256), the research project of the Administration of Traditional Chinese Medicine of Sichuan (No. 2016Q037), the Foundation of Science and Technology Agency of Chengdu (No. 2015-HM0100311-SF), the Fund of Antibiotics Research and Re-evaluation Key Laboratory of Sichuan Province, Sichuan Industrial Institute of Antibiotics, Chengdu University (No. ARRLKF17-10), and the Student Research Training Program (No. 201710613067).

\section{References}

[1] Zhou, S. L.; Liu, X. W.; Wu, L. Food. Fermn. Technol. 2011, 47, 98.

[2] Liu, D.; Fu, Z. X. Sci. Bull. 1985, 30, 239.

[3] You, Y. M. Chongqing Higher. Edu. Res. 2012, 31, 70.

[4] Liu, L. D.; Gu, W. J. Jiangxi Sci. 1992, 38.

[5] Yang, X. H. J. Hubei Institute for Nationalities (Nat. Sci). 2014, 282.

[6] Sun, H. L.; Wang, J. X.; Gu, X. Z. Chin. J. Exp. Tradit. Med. Form. 2011, 17, 94.

[7] Yang, D. Y.; Wang, F. S. Chin. Med. Mat. 1999, 295.

[8] Li, F.;You, Y. M. China Condiment. 2016, 41, 66.

[9] Chang, Y. C.; Chen, C. Y.; Chang, F. R. J. Chin. Chem. Soc-taip. 2001, 48, 811 .

[10] Chang, Y.; Chang, F.; Wu, Y. J. Chin. Chem. Soc-taip. 2013, 47, 373.

[11] Liu, T.; Li, W. Y.; Liu, X. W. Chin. Med. Mat. 2016, 39, 1789.

[12] Huh, G. W.; Park, J. H.; Shrestha, S. J.Appl. Biol. Chem. 2011, 54 , 309.

[13] Huh, G. W.; Park, J. H.; Kang, J. H. Nat. Prod. Res. 2014, 28, 831.

[14] Zhao, L. Q. Lishizhen Med. Mater. Med. Res. 2006, 17, 171.

[15] Suh, W. S.; Kim, K. H.; Kim, H. K. Cheminform 2015, 98, 1087.

[16] Yu, J. S.; Moon, E.; Kim, K. H. Bioorg. Chem. 2017, 74, 122.

[17] Seki, K.; Sasaki, T.; Haga, K. Phytochemistry 1994, 36, 949.

[18] Zhang, X. Y.; Tan, W. Q.; Yu, L. X. Prog. Mod. Biomed. 2010, 10, 133.

[19] Wang, R.; Tang, S. G.; Zhai, H. Y. Chin. J. Chin. Mater. Med. 2011, 36,1032 .

[20] Zhang, R. Y.; Zhao, D. H.; Sheng, B. H. Med. Dispute 1985, 200

[21] Cao, N. F.; Wu, X. H.; Kang, W. Y. Fine Chem. 2010, 27, 546.

Received March 2, 2018

Accepted March 25, 2018 